\title{
Sex differences in QEEG in adolescents with conduct disorder and psychopathic traits
}

\author{
Ana Calzada-Reyes ${ }^{1}$, Alfredo Alvarez-Amador ${ }^{2}$, Lidice Galán-García ${ }^{3}$, Mitchell Valdés-Sosa ${ }^{4}$ \\ ${ }^{1}$ Department of Clinical Neuroscience, Cuban Center for Neuroscience, Havana, Cuba \\ 2Department of Clinical Neurophysiology, Cuban Center for Neuroscience, Havana, Cuba \\ ${ }^{3}$ Department of Neurostatistic, Cuban Center for Neuroscience, Havana, Cuba \\ ${ }^{4}$ Department of Cognitive Neuroscience, Cuban Center of Neuroscience, Havana, Cuba
}

Received: May 15, 2018

Revised: December 5, 2018

Accepted: December 17, 2018

\section{Correspondence to}

\section{Ana Calzada-Reyes}

Department of Clinical Neuroscience, Cuban Center for Neuroscience, 190 street between 25th and 27th Avenue, Playa, Havana, Cuba

Tel: +53-7-263-7100

E-mail: anacalz@infomed.sld.cu

\section{ORCID}

\section{Ana Calzada-Reyes}

http://orcid.org/0000-0003-0082-2963

Alfredo Alvarez Amador

http://orcid.org/0000-0002-4549-3123

Lidice Galán-García

http://orcid.org/0000-0001-6400-6442

Mitchell Valdés-Sosa

http://orcid.org/0000-0002-1580-8374

Background: Sex influences is important to understand behavioral manifestations in a large number of neuropsychiatric disorders. We found electrophysiological differences specifically related to the influence of sex on psychopathic traits.

Methods: The resting electroencephalography (EEG) activity and low-resolution brain electromagnetic tomography (LORETA) for the EEG spectral bands were evaluated in 38 teenagers with conduct disorder (CD). The 25 male and 13 female subjects had psychopathic traits as diagnosed using the Antisocial Process Screening Device. All of the included adolescents were assessed using the Diagnostic and Statistical Manual of Mental Disorders, Fourth Edition, Text Revision (DSM-IV-TR) criteria. The visually inspected EEG characteristics and the use of frequency-domain quantitative analysis techniques are described.

Results: Quantitative EEG (QEEG) analysis showed that the slow-wave activities in the right frontal and left central regions were higher and the alpha-band powers in the left central and bitemporal regions were lower in the male than the female psychopathic traits group. The current source density showed increases in paralimbic areas at $2.73 \mathrm{~Hz}$ and decreases in the frontoparietal area at $9.37 \mathrm{~Hz}$ in male psychopathics relative to female psychopathics.

Conclusions: These findings indicate that QEEG analysis and techniques of source localization can reveal sex differences in brain electrical activity between teenagers with CD and psychopathic traits that are not obvious in visual inspections.

Key words: Electroencephalogram; Conduct disorder; Sex 


\section{INTRODUCTION}

Sex differences in the brain and behavior are among the most controversial topics in neuroscience. There is evidence for sex differences in the incidence and/or nature of several neuropsychiatric disorders. ${ }^{1}$ A compelling reason for studying sex differences in the brain is that the onset and clinical manifestations of almost all neuropsychiatric illnesses differ between males and females. ${ }^{2}$ Conduct disorder (CD), ${ }^{3}$ attention deficit and hyperactivity disorder (ADHD), ${ }^{4,5}$ and oppositional defiant disorder (ODD) ${ }^{6,7}$ are significantly more common in males.

One form of CD is distinguished by the presence of psychopathic traits. The disorder interferes with numerous aspects of normal development and functioning in a teenager's life, and predisposes the adolescent to a greater degree of psychiatric and social disorders in later life. The psychopathic phenotype clearly differs between males and females, with the prevalence of psychopathic traits being lower ${ }^{8}$ and the onset being later in females, and the developmental course being different, ${ }^{8,9}$ as is also the prototypical manifestation, with less aggressive behavior among females than males. ${ }^{8,9}$ The study and recognition of psychopathic traits in childhood and adolescence could provide a better understanding of the etiology of the disorder and facilitate the development of effective therapeutic interventions. ${ }^{10-12}$ Some electroencephalography (EEG) studies have revealed brain functional sex differences in control subjects and in patients with certain psychiatric disorders.

The following characteristics have been found:

1) The absolute EEG powers in the delta, theta, and beta bands were higher in females than in males. ${ }^{13}$

2) The sex differences in EEG activity were more pronounced during photic stimulation, and the coherence in the theta band was higher in females than in males. ${ }^{14}$

3) Healthy control females showed greater interhemispheric coherences than control males in the gamma band. ${ }^{15}$

4) Females showed higher relative EEG spectral power in the alpha band and lower power in the theta band. Additionally, the theta/alpha ratio was significantly lower for females than for males. ${ }^{16}$

5) No frontal brain asymmetry was found at 4.5 years of age, and no left frontal activation was found at 8 years in healthy female. In contrast, healthy male demonstrated greater right than left frontal activation, whereas preschool boys with ODD did not display any frontal brain asymmetry. ${ }^{17}$

6) Quantitative EEG (QEEG) studies have revealed increased widespread theta-band activity in adolescent males with ADHD, whereas ADHD females showed localized frontal enhancement in the theta band. ${ }^{18}$ Boys with ADHD showed higher absolute and relative theta-band powers, higher theta/beta ratio, lower absolute and relative alpha-band powers, and lower absolute and relative beta-band powers compared with controls with ADHD. Girls with ADHD had higher absolute delta-band power, higher absolute and relative theta-band powers, higher theta/beta ratio, higher total power, and lower relative delta- and beta-band powers compared with control girls. ${ }^{18}$

These results will help in understanding the mechanisms underlying sex differences in neuropsychiatric disorders, which can in turn lead to the establishment of effective preventive and therapeutic strategies. Sex differences in psychopathic traits constitute an important field of neuroscience research, but there has been a sparsity of investigations into this topic, especially in teenagers. We have previously reported on EEG abnormalities in a group of 25 male teenagers suffering from CD and psychopathic traits. ${ }^{19}$ That psychopathic traits group had higher beta-band power in the bilateral frontal-temporal regions and lower alpha-band power in the left central-temporal and right frontal-central-temporal regions than the control group. Low-resolution brain electromagnetic tomography (LORETA) showed differences especially in the fronto-temporo-striatal areas.

Here we present results obtained from a larger group of $C D$ and psychopathic traits teenagers of both sexes. The aim of this study was to identify any electrophysiological differences between male and female teenagers with $C D$ and psychopathic traits, in particular to determine whether obtained QEEG and LORETA results are influenced by sex.

\section{MATERIALS AND METHODS}

\section{Subjects}

This study included 38 male and female CD teenagers aged 
$15.50 \pm 0.21$ years (mean \pm standard deviation) attending a special CD school located in Havana City. The assessments were conducted during a 2-year period from January 2010 to December 2011.

Clinical assessments were performed by child psychiatrists who were directly involved in this study. All of them were well trained in the application of all of the structured and semistructured interviews and diagnostic tools utilized. The diagnostic interviews were carried out separately with participants and caregivers. The clinical interviews incorporated information from as many sources as possible, including the history as provided by a parent or guardian, school reports, data from any other health professionals, and behaviors observed during the assessments.

This study used DSM-IV-TR ${ }^{20}$ criteria to diagnose CD, and the Antisocial Process Screening Device (APSD) to evaluate psychopathic traits. ${ }^{21}$ The 25 male adolescents aged 15.66 \pm 1.21 years and the 13 female adolescents aged $15.35 \pm$ 0.66 years had all been diagnosed with CD and psychopathic traits. In order to be included in the CD with comorbid psychopathic traits group, adolescents were required to meet the full diagnostic criteria for psychopathic disorder according to the APSD scale created by Frick and Hare. ${ }^{21}$ This scale is an extension of the Psychopathy Checklist-Revised for adults. ${ }^{22}$ The APSD is a 20-item behavior rating scale that is typically completed by a parent and/or teacher, and it does not specify a particular amount of time that the rater needs to have known the child. The APSD was designed to assess a lack of empathy and callous behavior toward others, which may reflect a distinct subtype of those with more severe externalizing behavior problems and worse developmental outcomes. Factor analysis of the APSD uncovered three dimensions: narcissism, impulsivity, and callousunemotional (CU). The CU dimension includes items such as "concerned about the feelings of others" and "does not show emotions."12

Sociobiographical, criminological, medical, and psychopathological information was recorded on a standardized score sheet and entered into a computer system for further processing. Exclusion criteria in this study were the presence of any current or previous history of neurological disease that can influence EEG recording, the presence of an abnormal prenatal, perinatal, or neonatal event, disorder of consciousness, head injury resulting in cognitive deficits, antecedents of central nervous system (CNS) diseases, convulsions, psychiatric abnormalities (other than $C D, A D H D$, or ODD), or substance abuse disorders. None of the included adolescents was taking any medication at the time of testing.

\section{EEG procedure}

EEG was performed using a 21-channel digital EEG hardware and software package (MEDICID IV, Neuronic, Havana, Cuba). Surface electrodes were placed at 19 sites (Fp1, Fp2, $F Z$ F3, F4, F7, F8, CZ, C3, C4, T3, T4, T5, T6, Pz, P3, P4, O1, and $\mathrm{O} 2$ ) according to the international 10-20 system, and they were referenced to linked earlobes with a ground electrode attached to the forehead. The impedance of all electrodes did not exceed $5 \mathrm{k} \Omega$ throughout the recordings. EEG signals were amplified 10,600-fold, bandpass filtered from 0.5 to 30 $\mathrm{Hz}$, and sampled by a 12-bit analog-to-digital converter at $200 \mathrm{~Hz}$.

The electrodes were placed while subjects were familiarizing themselves with the testing equipment and procedures. EEG recordings were made in a quiet air-conditioned room with the experimenter and recording equipment present. All subjects were instructed to relax and remain still during testing in order to minimize artifacts produced by ocular movements, and to avoid excessive blinking. During the recordings the subjects were seated on a reclining chair awake with their eyes closed. All recordings were performed during the morning.

Resting EEG was performed for 8-10 minutes with the eyes closed, which was followed by 2 minutes of alternation between closed and opened eyes, 3 minutes while hyperventilating, and 2 minutes of recovery. Taking into account that sleepiness might have enhanced the theta-band activity, the individual vigilance level was checked during EEG recordings to monitor for slowing of the EEG background activity or the appearance of typical sleep patterns. In addition, at the end of the recording process, individuals were asked whether they were awake throughout the recording process. Participation in the study was voluntary, and written informed consent was obtained from all of the parents. The study was approved by the Ethics Committee of the Cuban Center for Neuroscience. 


\section{Visual EEG assessments}

A clinical neurophysiologist (the first author) performed and interpreted the EEG recordings. Several bipolar montages were used for off-line EEG data interpretation. An EEG recording was considered normal if it presented an adequate organization of the background activity (in accordance with the subject's age), well-defined spatial differentiation, rhythmic alpha-band activity, and the absence of paroxysmal activity. The slow subgroup was characterized by the presence of persistent nonrhythmic theta- and delta-band slow waves. The paroxysmal EEG category included recordings with graphoelements with sudden onset, rapid attainment of a maximum and abrupt termination, and distinguished from background activity such as a spike, a sharp wave, and a spike and wave. A spike is by convention defined as a waveform with a duration between 20 and 70 msec. Paroxysmal activity that lasts 70-200 msec is referred to as a sharp wave and spike, and a wave complex if one spike is followed by a delta-band wave. EEG recordings with both types of the above-described abnormalities were allocated to the slow and paroxysmal category. Ratios and percentages were calculated in all categories.

\section{QEEG analysis}

Tracings were visually inspected and edited off-line in order to eliminate epochs with movement artifacts, eye blinking, muscle activity, or drowsiness. For each subject, 20-24 EEG segments out of a total of 32 artifact-free recordings lasting 2.56 seconds in the closed-eyes state were selected. Spectral analysis was implemented using the fast Fourier transform to obtain the estimated cross-spectral matrices in all individual records. ${ }^{23}$ Cross-spectral matrices were calculated for every $0.39 \mathrm{~Hz}$ from 0.78 to $19.50 \mathrm{~Hz}$.

\section{EEG source estimation}

The EEG tomography was conducted using LORETA. ${ }^{24}$ Each LORETA file was computed from the frequency-domain EEG cross-spectra corresponding to the selected EEG frequencies, one within the alpha band $(9.76 \mathrm{~Hz})$, the second within the delta band $(2.73 \mathrm{~Hz})$, and another within the theta band $(4.68 \mathrm{~Hz})$. These three specific frequencies were selected taking into consideration the results from comparing between the $Z$ cross-spectra of the two groups (see below).

Current density vectors (CDV) were calculated for each in- dividual from all of the data segments using the Source Localizer software (Neuronic). The lead field (LF) was computed first in the brain electrical tomography analysis. This software computes the electrical LF using the isotropic and piecewise homogeneous three-spheres head model. In the present research, the distribution of the current density inside the head was calculated using a three-dimensional grid that encompassed the entire brain volume. Anatomical information for LF computation was provided by a brain average template obtained from the Montreal Neurological Institute. In this study the predefined source space was cortical gray matter plus basal ganglia, and utilized $4 \times 4 \times 4$ grids. There were 21,926 generators used. The obtained tomography data were visualized using the Tomographic Viewer software (Neuronic).

\section{Statistical analysis}

The dependence among categorical variables (demographic, behavior, and EEG visual inspections) was determined using the Pearson Chi-square test and one-way ANOVA test (Statistica ver. 6.0 for Windows; Dell, Santa Clara, CA, USA). The threshold for statistical significance was 0.05 in all of the tests.

\section{QEEG spectrum analysis}

The Cuban normative database was used to compare the psychopathic male traits group and psychopathic female traits group with normative values. Szava et al. ${ }^{25}$ reported the methodology for constructing the normative database in 1994. As much of the life span as possible was included in the normative sample (age range of 5 to 97 years). Careful screening of a random sample from the 116,000 inhabitants of Havana was applied using stringent inclusion criteria, which resulted in $65 \%$ of the subjects being rejected. The age range under investigation was divided into quasilogarithmically spaced intervals (yearly from 5 to 15.9 years, every 2 years from 16 to 19.9 years, and every 5 years from 20 to 97 years) in order to cope with growth spurts. This schema generated a normative sample of 211 functionally healthy subjects (105 males and 106 females). EEG was performed using the same conditions in each subject. The $Z$ standardized statistic was calculated for all spectral parameters according to 


$$
z=\frac{x-\mu \text { (age) }}{\sigma \text { (age) }}
$$

where $\mu$ and $s$ are the mean and SD, respectively, estimated in the normative group for the specific age of the subject been compared. The value of $\mathrm{x}$ is the observation made for each subject.

\section{Statistical methodology to compare the $Z$ spectra means of the two groups}

A permutation test was used to evaluate differences between the Z spectra of the two groups. ${ }^{26,27}$ Using a permutation test provides the following advantages: free distribution, which controls the experiment-wise error for simultaneous univariate comparisons, no assumption of an underlying correlation structure, and providing exact probability values for any numbers of individuals, frequency points, and recording sites.

Multivariate statistics were used to summarize and test differences between two Z spectra obtained from the nonparametric combination of dependent permutation tests, which is commonly applied when there is a finite number of multivariate tests. These multivariate statistics are calculated according to a combined univariate $t$-test. The combine function used was the maximum.

Two separate partial tests were performed:

1) The maximum of $t$ statistic at each electrode for all frequencies.

2) The maximum of $t$ statistic at each frequency for all electrodes.

These methodologies were implemented using the following steps:

1) The observations were repeatedly permutated be- tween groups. Both statistics were calculated for each repetition.

2) The empirical distribution was estimated using the statistics calculated in step 1 for all permutations.

3) Significance levels were set using the $t$ and $\max (t)$ values of the original samples in the empirical distribution estimated in step $2 .{ }^{26,27}$

\section{Inverse solution analysis}

A $t$-test for independent samples with correction for multiple comparisons was performed (Statistica, Neuronic) to identify significant regional differences between groups in CDV for the delta and alpha bands. The final outcome was a map of the $t$-test values for each voxel thresholded at false discovery rates (FDRs) of $q=0.2$ and $q=0.1^{28}$ to control for the increased risk of false positives due to the large number of statistical tests that were carried out simultaneously. This procedure operates simultaneously on all voxelwise test statistics to determine which tests should be considered to be statistically significant. The coordinates of main activation were represented in Talairach space (Tomographic Viewer; Neuronic).

\section{RESULTS}

\section{Demographic and behavior findings}

Analyses of demographic data indicated that age did not differ significantly between the two groups (Table 1). The ASPD scores showed significant group differences, whereas $A D H D, C D$, and ODD as a comorbidity with a CD diagnosis did not (Table 1).

Table 1. Comparison of demographic and behavior variables between the male and female teenagers with psychopathic traits

\begin{tabular}{lccc}
\hline Variable & Male psychopathic traits & Female psychopathic traits & Statistic \\
\hline Age (years) & $15.66(1.21)$ & $15.35(0.66)$ & $\mathrm{F}=0.40$ \\
ASPD score & $27.76(4.30)$ & $22.16(6.09)$ & $\mathrm{x}^{2}=0.00^{\mathrm{a}}$ \\
ADHD & 11 & 5 & $\mathrm{x}^{2}=0.41$ \\
Dissocial conduct disorder & 16 & 9 & $\mathrm{x}^{2}=0.76$ \\
Oppositional defiant disorder & 5 & 3 & $\mathrm{x}^{2}=0.83$
\end{tabular}

Values are presented as number (\%) unless otherwise indicated.

ADHD, attention deficit and hyperactivity disorder; APSD, antisocial process screening device.

${ }^{a}$ Anova or Pearson $x^{2}, p<0.05$. 


\section{EEG visual inspections}

The resting EEG visual analyses revealed a high percentage of EEG abnormalities in both of the studied groups. The EEG recordings were abnormal in 34 (89.5\%) of the adolescents: $24(96.0 \%)$ of those in the male psychopathic traits group and 10 of those (76.9\%) in the female psychopathic traits group.

Table 2 presents the results of the EEG visual analysis, which produced very similar results in the male and female psychopathic traits groups. The paroxysmal EEG category had the largest number of subjects (around $42.1 \%$ ), followed by the slow EEG category (36.8\%). Eight adolescents from both groups were included in the other two categories. The Pearson Chi-square test comparison did not reveal significant differences between the two groups regarding the total EEG recordings with abnormalities versus normal EEG recordings by visual inspection $\left(x^{2}=3.72, \mathrm{df}=3, p=0.31\right.$ ).

Table 3 presents the topographic distribution of the EEG abnormalities found in both groups. The frontal lobe was the single brain region most affected by EEG abnormalities in both groups. A comparison using the Pearson Chi-square test was made while considering only the paroxysmal EEG category (because it was the EEG abnormality that occurred most frequently in both groups) and comparing frontal versus temporal localizations. There were no differences between these topographic distributions of paroxysmal EEG activity between the two groups $\left(x^{2}=3.41, \mathrm{df}=1, p=0.06\right)$. Paroxysmal EEG activity was more frequent in the frontal area in the male psychopathic traits group, while in the female group this EEG abnormality was equally present in both localizations.

\section{QEEG analysis}

The duration of the EEG recordings in the closed-eyes state was $50.08 \pm 21.86$ seconds for the male psychopathic traits CD group and $60.07 \pm 18.41$ seconds for the female psychopathic traits CD group ( $p=0.43$ ). Statistically significant differences in the mean parameters of cross-spectral measures between the male and female psychopathic traits CD groups using the permutation test were found in the delta band at $1.56-2.73 \mathrm{~Hz}$ in the right frontal area, and in the theta band at $4.68-6.63 \mathrm{~Hz}$ in the left central area. The power values for these frequencies were increased for the male psychopathic traits group, whereas in the alpha band at 9.36-10.10 Hz, a decrease in the power was found in the left central and bilateral temporal regions for this group.

\section{EEG source analysis}

\section{Comparison of delta-band activity between groups}

The delta-band activity $(2.73 \mathrm{~Hz})$ in the LORETA source imaging was higher in the male than the female psychopathic traits CD group, specifically in Brodmann areas 6, 11, 24, 25, $28,34,37,38$, and 47 (orbitofrontal cortex, supplementary motor area, fusiform area, rectus area, olfactory area, anterior cingulate, medial cingulate, medial temporal pole, parahippocampus, and amygdala) in both hemispheres; in Brodmann areas 8, 13, 21, and 38 (insula, superior temporal pole, medial temporal gyrus, and superior frontal gyrus) in the right hemisphere; and in Brodmann area 35 (hippocampus)

Table 2. Classification of the adolescent electroencephalograms (EEG) in both groups by visual inspection

\begin{tabular}{lcccc}
\hline Group & Normal & Slow & Paroxysmal & Slow and paroxysmal \\
\hline Male psychopathic traits & $1(4.0)$ & $9(36.0)$ & $12(48.0)$ & $3(12.0)$ \\
Female psychopathic traits & $3(23.1)$ & $5(38.5)$ & $4(30.8)$ & $1(7.7)$ \\
\hline
\end{tabular}

Values are presented as number (\%).

Table 3. Topographic distribution of electroencephalography (EEG) abnormalities

\begin{tabular}{|c|c|c|c|c|c|c|c|}
\hline \multirow{2}{*}{ Group } & \multicolumn{3}{|c|}{ Slow } & \multicolumn{2}{|c|}{ Paroxysmal } & \multicolumn{2}{|c|}{ Slow and paroxysmal } \\
\hline & Frontal & Temporal & Widespread & Frontal & Temporal & Frontal & Widespread \\
\hline Male psychopathic traits & $6(24)$ & $0(0)$ & $3(12)$ & $11(44)$ & $1(4)$ & $1(4)$ & $2(8)$ \\
\hline Female psychopathic traits & $2(15.4)$ & $1(7.7)$ & $2(15.4)$ & $2(15.4)$ & $2(15.4)$ & $1(7.7)$ & $0(0)$ \\
\hline
\end{tabular}

Values are presented as number (\%). 
in the left hemisphere (FDR q = 0.2) (Table 4, Fig. 1).

\section{Comparison of alpha-band activity between groups}

The alpha-band activity $(9.36 \mathrm{~Hz})$ in the LORETA source imaging was lower in the male than the female psychopathic traits CD group, specifically in Brodmann areas 2, 6, 7, 8, 13, $39,40,43,44,45,46$, and 47 (orbitofrontal cortex, three parts of the inferior frontal gyrus, medial frontal gyrus, opercular parts of the inferior frontal gyrus, superior frontal gyrus, insula, precentral area, supplementary motor area, postcentral area, supramarginal area, superior parietal gyrus, inferior parietal gyrus, and angular gyrus) in the left hemisphere (FDR $q=0.1$ ) (Table 5, Fig. 2). There were no significant group differences in the theta band.

\section{DISCUSSION}

Frontal slow-wave and paroxysmal activities were the abnormalities identified the most frequently in both groups by

Table 4. Brain areas showing significant differences $(q=0.2)$ within the delta band $(2.73 \mathrm{~Hz})$ between male and female teenagers with psychopathic traits according to a voxel-by-voxel analysis using low-resolution brain electromagnetic tomography (LORETA)

\begin{tabular}{|c|c|c|c|c|}
\hline$x-m m$ & $y-m m$ & z-mm & Brodmann area & Anatomical location \\
\hline 15 & 31 & -24 & Brodmann area 11 & Orbitofrontal cortex \\
\hline-18 & 14 & -24 & Brodmann area 11 & Orbitofrontal cortex \\
\hline 37 & 15 & -8 & Brodmann area 47 & Orbitofrontal cortex \\
\hline 8 & 25 & 52 & Brodmann area 6 & Supplementary motor area \\
\hline-5 & -12 & 52 & Brodmann area 6 & Supplementary motor area \\
\hline 25 & -10 & 60 & Brodmann area 8 & Superior frontal gyrus \\
\hline 27 & 12 & -44 & Brodmann area 37 & Fusiform \\
\hline-21 & 2 & -44 & Brodmann area 37 & Fusiform \\
\hline-20 & -9 & -20 & Brodmann area 35 & Hippocampus \\
\hline 45 & -9 & -28 & Brodmann area 38 & Superior temporal pole \\
\hline 42 & 19 & -44 & Brodmann area 38 & Medial temporal pole \\
\hline 31 & 11 & -8 & Brodmann area 13 & Insula \\
\hline 20 & -4 & -28 & Brodmann area 28 & Parahippocampal \\
\hline-18 & -5 & -28 & Brodmann area 28 & Parahippocampal \\
\hline-20 & -6 & -16 & Brodmann area 34 & Amygdala \\
\hline 28 & -1 & -28 & Brodmann area 34 & Amygdala \\
\hline-6 & 29 & -20 & Brodmann area 11 & Rectus \\
\hline 8 & 23 & -24 & Brodmann area 11 & Rectus \\
\hline 13 & 8 & -16 & Brodmann area 25 & Olfactory \\
\hline-11 & 9 & -16 & Brodmann area 25 & Olfactory \\
\hline 6 & 22 & 32 & Brodmann area 24 & Medial cingulate \\
\hline-5 & 22 & 36 & Brodmann area 24 & Medial cingulate \\
\hline 52 & 1 & -28 & Brodmann area 21 & Medial temporal \\
\hline 5 & 11 & 24 & Brodmann area 24 & Anterior cingulate \\
\hline-4 & 21 & 24 & Brodmann area 24 & Anterior cingulate \\
\hline-24 & 17 & -36 & Brodmann area 38 & Medial temporal pole \\
\hline
\end{tabular}

Coordinates are given in millimeters, and the origin is at the anterior commissure. Negative and positive $x, y$, and $z$ values represent left and right, respectively, posterior and anterior, and inferior and superior. 


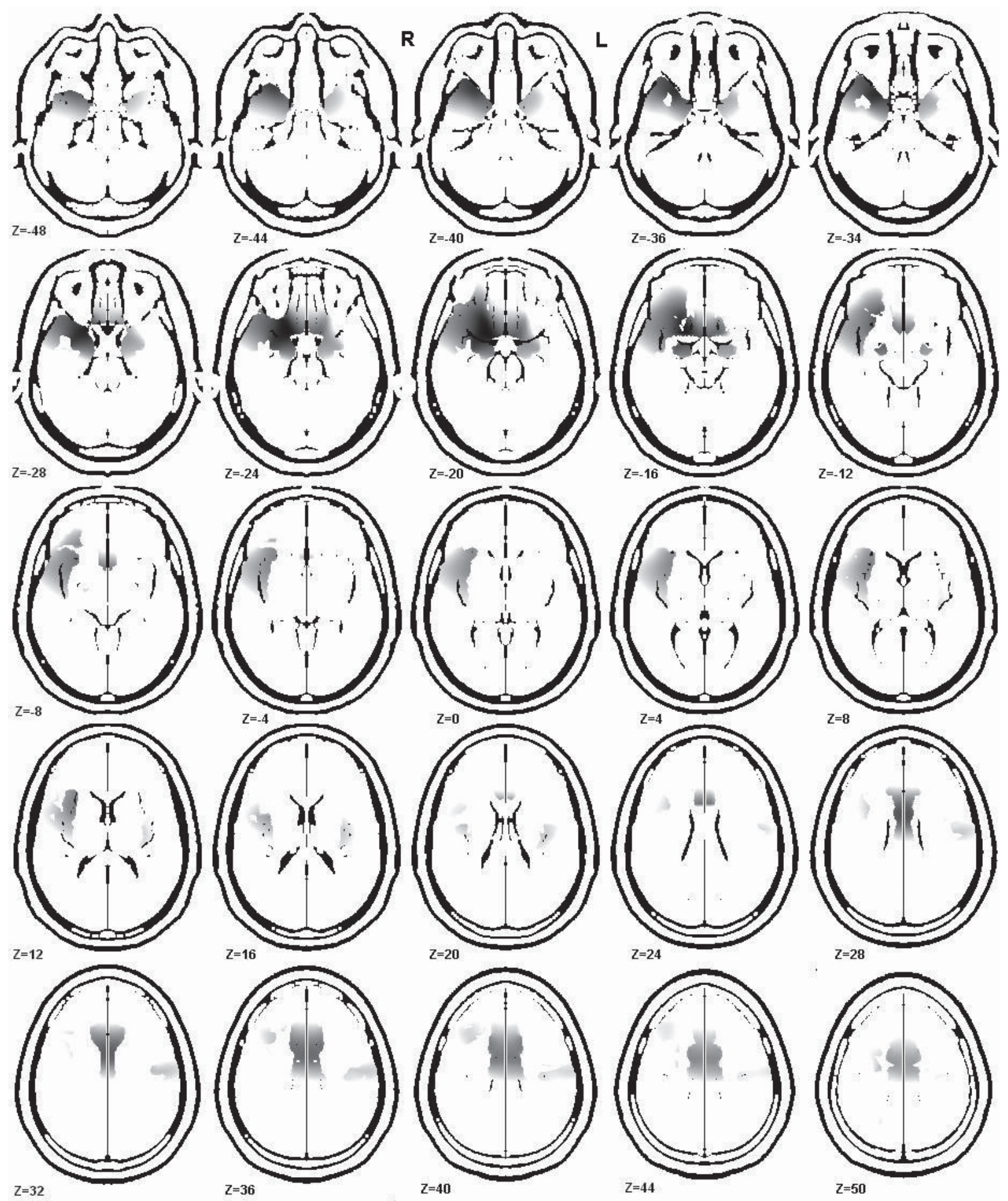

Fig. 1. The anatomical distribution of the sources of delta-band activity $(2.73 \mathrm{~Hz})$ that were increased in the male psychopathic traits group were localized over a broad region within both hemispheres. The main regions where the activity was higher in the male than the female psychopathic traits group were the orbitofrontal cortex, supplementary motor area, fusiform area, rectus area, olfactory area, anterior cingulate, medial cingulate, medial temporal pole, parahippocampus, and amygdala in both hemispheres; the insula, superior temporal pole, medial temporal gyrus, and superior frontal gyrus in the right hemisphere; and the hippocampus in the left hemisphere. $R$, right hemisphere; $L$, left hemisphere; $Z$, the $z$-axis value for each image of the shown brain template. 
Table 5. Brain areas showing statistical differences $(q=0.1)$ in alpha frequency $(9.36 \mathrm{~Hz})$ between male and female teenagers with psychopathic traits

\begin{tabular}{lccll}
\hline $\mathbf{x}-\mathbf{m m}$ & $\mathbf{y}-\mathbf{m m}$ & $\mathbf{z}-\mathbf{m m}$ & Brodmann area & \multicolumn{1}{c}{ Anatomical location } \\
\hline-46 & 30 & -8 & Brodmann area 47 & Orbitofrontal cortex \\
-53 & 27 & 12 & Brodmann area 45 & Tri portions of the inferior frontal gyrus \\
-15 & 34 & 52 & Brodmann area 8 & Superior frontal gyrus \\
-35 & 33 & 32 & Brodmann area 46 & Medial frontal gyrus \\
-55 & 6 & 12 & Brodmann area 44 & Opercular portions of the inferior frontal gyrus \\
-30 & 20 & 4 & Brodmann area 13 & Insula \\
-64 & 6 & 24 & Brodmann area 6 & Precentral \\
-58 & -1 & 2 & Brodmann area 43 & Postcentral area \\
-61 & -38 & 32 & Brodmann area 2 & Supramarginal \\
-10 & 19 & 60 & Brodmann area 8 & Supplementary Motor area \\
-26 & -55 & 60 & Brodmann area 7 & Superior parietal gyrus \\
-40 & -50 & 56 & Brodmann area 40 & Inferior parietal gyrus \\
\hline-42 & -62 & 64 & Brodmann area 39 & Angular gyrus \\
\hline
\end{tabular}

According to voxel by voxel analysis with Low-Resolution Brain Electromagnetic Tomography (LORETA). Coordinates are given in millimeters, and the origin is at the anterior commissure. For $x$, negative values represent left, positive values represent right. For $y$, negative values represent posterior, positive values represent anterior. For $z$, negative values represent inferior, positive values represent superior.

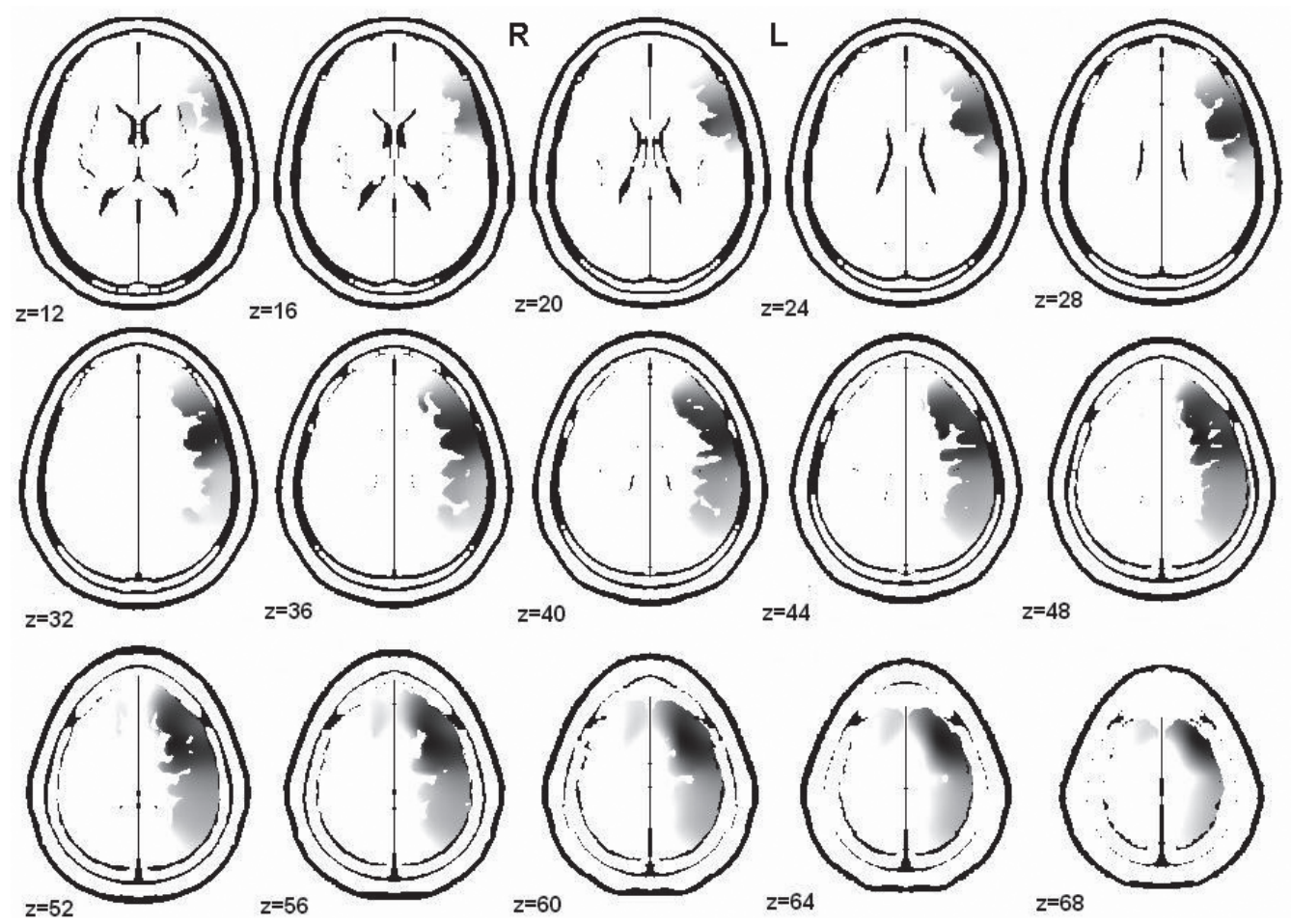

Fig. 2. The anatomical distribution of the sources of alpha-band activity $(9.36 \mathrm{~Hz})$ that were decreased in the male psychopathic traits group were localized over a broad region within the left hemisphere. The main regions where the activity was lower in the male than the female psychopathic traits group were the orbitofrontal cortex, three parts of the inferior frontal gyrus, medial frontal gyrus, opercular parts of the inferior frontal gyrus, superior frontal gyrus, insula, precentral area, supplementary motor area, postcentral area, supramarginal area, superior parietal gyrus, inferior parietal gyrus, and angular gyrus in the left hemisphere. $R$, right hemisphere; L, left hemisphere; Z, the Z-axis value for each image of the shown brain template. 
visual inspection of the EEG recordings. No significant differences were found between the male and female groups in the presence of abnormalities or the localization of the paroxysmal EEG activity.

EEG studies of adolescents with CD, aggressive children, and teenagers with $C D$ and psychopathic traits have found similar frequencies of EEG abnormalities in qualitative EEG assessments to those found in the present study. ${ }^{29-32}$ Nonetheless, the previously reported findings have been mainly based on male or mixed-sex samples. Significant differences in QEEG and regional current source densities were found between the present male and female psychopathic traits groups. The slow-wave powers in the right frontal and left central regions were higher and the alpha-band activities in the left central and bitemporal regions were lower in the male than the female psychopathic traits group. LORETA revealed differences especially in the fronto-temporo-limbic and parietal regions.

QEEG revealed that the slow-wave activities were higher and the alpha power was lower in the male than the female psychopathic traits group. Nevertheless, sex differences have also been reported in normal children, with males having lower theta-band power and higher alpha-band power than females. ${ }^{33}$ Other authors studying children, adolescents, and adults with $\mathrm{ADHD}^{5,33}$ and antisocial offenders ${ }^{34}$ have reported similar results.

Recent research suggests oscillations in brain waves reflect a mechanism of cerebral integration. Oscillations in delta-band in humans are prominent only during the early developmental stages and slow-wave sleep. There is evidence of delta-band activity being increased in almost all pathological states associated with brain tissue damage, developmental disorders, or even more subtle disorders without known organic causes. ${ }^{35}$ High tonic theta-band EEG power could reflect a deficiency in the information processing capacity of the CNS. ${ }^{36}$ Slow EEG activity (theta and delta bands) has been strongly correlated with cholinergic activity and central cholinergic pathways. In vitro studies have revealed that acetylcholine can either inhibit or excite cortical pyramidal neurons. The production of inhibition results from the excitation of the intrinsic inhibitory neurons in the cortex. The increased slow-wave EEG activity in male adolescents may indicate an imbalance in excitation-inhibition homeostasis in the cortex.
Decreased alpha-band activity in adolescents with CD has been ascribed to a maturational retardation hypothesis. ${ }^{37}$ Lower alpha-band activity has been observed in delinquent children, institutionalized children, ${ }^{38}$ and psychopathic offenders. ${ }^{34}$ In neurologically intact subjects, the power in the alpha band increases from early childhood to adulthood, and the adult pattern of dominant alpha-band rhythm becomes evident in late adolescence and is referred to as being characteristic of mature EEG findings. Our findings support that functional cortical development is more impaired in male than female adolescents with CD and psychopathic traits.

Modulations in the alpha band depend on the structural integrity of white-matter tracts (WM). ${ }^{39} \mathrm{~A}$ longitudinal MRI study of child and adolescent brain development found sex differences in the rate of linear WM increase, with age-related increases being greater in males than females. This WM increase makes signal transduction faster and more efficient, and unsuccessful signal transduction due to reduced myelination could explain the reduction in alpha-band power detected in male psychopathic adolescents in the present study. A diminished alpha-band activity associated with increased slow-wave activity could lead to the greater cognitive deficits and the lack of inhibitory control exhibited by male adolescents with $C D$ and psychopathic traits compared with females with the same psychiatric diagnosis. It has also been shown that oscillations in slow and alpha-band waves are reciprocally related to each other. This reciprocal relationship might reflect an inhibitory control over motivational and emotional drives, which is implemented by the prefrontal cortex. There is some evidence that increases in slowwave EEG activity in adolescence predict the emergence of antisocial behavior later in life.

LORETA is a functional source imaging method with certain electrophysiological and neuroanatomical constraints. ${ }^{24}$ The LORETA algorithm solves the inverse problem by assuming specific orientations and strengths of neighboring neuronal sources (represented by adjacent voxels). LORETA has been recognized as an efficient tool for functional mapping since it is consistent with physiology and can be used for localization.

LORETA is a noninvasive source localization neuroimaging method with a high temporal resolution that facilitates a deeper understanding of the neuropsychiatry revealed by 
EEG recordings. The present LORETA findings have helped to define the structures related to the increase in delta-band activity and the decrease in alpha-band activity as a sign of dysfunctional (behavior disinhibition) neuronal state, possibly secondary to brain damage. To our knowledge, no previous studies have used LORETA to demonstrate differences in brain function between male and female adolescents with $\mathrm{CD}$ and psychopathic traits.

The present LORETA and surface EEG findings are also consistent with MRI studies showing reductions in the fronto-temporo-limbic and parietal lobe volumes, ${ }^{40}$ reduced cortical thickness in male adolescents with $C D$, and varying levels of CU traits. ${ }^{41}$ Dysfunction in the limbic and paralimbic circuitry when making moral judgments has been observed in male adolescents with psychopathic traits causing deficits in emotional processing.

Furthermore, there is evidence linking atypical functioning in the fronto-temporal and paralimbic regions with impairments in social cognition, affect processing and regulation, and moral decision-making, as often observed in adolescents with psychopathic traits. ${ }^{42}$ Areas of the prefrontal cortex are important for the generation of response inhibition and self-control, which are key components of emotion regulation. ${ }^{43}$

Interesting and reproducible relationships have been found between current sources and network connectivity that provide a deeper understanding of the surface EEG dynamics. ${ }^{44}$ Psychopathy has been associated with connectivity among brain regions known as the "default mode network," which includes the medial prefrontal cortex, posterior cingulate, and inferior parietal lobule; frontoparietal connectivity; and a visual-posterior cingulate connectivity. ${ }^{45}$ According to reports on sex differences of brain connectivity, our findings could be reflecting abnormal functional connectivity among the brain structures related to the increased delta-band activities and reduced alpha-band activities in male adolescents.

There are emotional and behavior differences between females and males with psychopathic traits. Some research indicates that girls with disruptive behavior are more likely than boys with same diagnosis to behave covertly, lie, and engage in nonviolent crimes and sexually promiscuous behaviors. Female teenagers with these traits generally show fewer CU traits, more emotional symptoms, more prosocial behaviors, and lower crime seriousness compared with males with the same diagnosis. Our electrophysiological findings could be related to sexual dimorphism in development or to activation of different underlying neural circuitry in males compared with female adolescents with this psychiatry diagnosis.

Our findings support the hypothesis that impairment of the paralimbic system including the cortical and subcortical regions contributes to the genesis of psychopathic behavior. ${ }^{46}$ The paralimbic areas participate in emotional processing, attention control, and behavior inhibition. Even though recent studies have found that the volume of paralimbic regions is reduced in adolescents of both sexes with psychopathic traits, the current results indicate that the functional alterations are greatest in males. Our results are also consistent with the neurodevelopmental disorder perspective of psychopathy proposed by Gao et al, ${ }^{47}$ in which the disorder has its origins early in life.

Disruption of the processing of punishment information is the key element of the emotion theoretical hypothesis for explaining the psychopathy. Blair considered that both the ventromedial prefrontal cortex and amygdala are involved, ${ }^{48}$ whereas Kieh $l^{49}$ implicated the paralimbic and limbic systems. Our findings suggest that deficits in decision-making, behavior control, and emotional regulation are more marked in males than female adolescents with psychopathic traits. These features could be at least partly due to functional anomalies in the fronto-temporo-limbic and parietal regions. Dysfunction of the superior parietal lobes could originate from deficits in the processing of sensory information and anomalous judgments of both the world and one's own body, resulting in abnormal emotional responses in individuals with psychopathic traits. ${ }^{50}$ Fronto-temporo-limbic regions are important for linking the evolutionarily newer cortical regions responsible for cognition and behavior with older structures responsible for emotion and visceral states. Our research confirms that functional impairments of the CNS in male adolescents with psychopathic traits involve more than one brain region.

Recent MRI results suggest that abnormalities in the structure and function of limbic areas and the prefrontal cortex in adolescents with CU traits should not be viewed in isolation. ${ }^{51}$ Reduced connectivity between the amygdala and prefrontal areas was reported in adolescents with 
CD compared with controls while watching displays of deliberately inflicted pain. Other studies employing diffusion-tensor imaging found reduced fractional anisotropy of the uncinate fasciculus pathway connecting the amygdala and orbitofrontal cortex in adolescents with $C D .^{52}$ Structural neuroimaging studies have shown decreases in the volume of gray matter in the prefrontal cortex, insula, and paralimbic regions in adolescents with psychopathic traits. ${ }^{51,53}$

In summary, the results of this study support the hypothesis that brain functioning in the fronto-temporo-limbic and parietal areas are impaired in adolescents with psychopathic traits. QEEG and LORETA have revealed sex differences between adolescents with CD and psychopathic traits in terms of their EEG profiles. Both types of EEG assessment could be important tools for recognizing functional abnormalities of the CNS that could be useful when designing behavior or therapeutic strategies for children with psychopathic traits or risk factors for this psychiatric diagnosis.

The current study was subject to the following limitations. First, we only used EEG visual inspections to remove artifacts. However, such a manual rejection process has the advantage of not being computationally demanding, and it can be assumed that a human expert would be able to identify all of the artifact-contaminated epochs and remove them from the analysis. However, the procedure of selecting the artifact-free trials may be subjective, and so future studies should use automated methods to remove residual artifacts not detected by visual inspection. Second, there are some reports of sex and handedness differences in EEG measures of hemispheric specialization, and so it would be advisable for future research to include evaluations of handedness preference and its possible connection with the presence of lateralized abnormalities when comparing sex influences in the $C D$ and psychopathic traits of teenager groups.

This study has revealed significant sex differences in the activities in the theta and alpha bands in QEEG parameters in teenagers with CD and psychopathic traits. We have also provided evidence of significant differences in brain function in the fronto-temporo-limbic and parietal regions as revealed by LORETA between the two groups studied. These results are consistent with those obtained in other structural and functional imaging studies of male adolescents, and provide evidence that male adolescents with CD and psychopathic traits show greater functional impairment as detected by QEEG in brain regions implicated in emotion processing and empathy compared to females with the same psychiatric diagnosis.

\section{Conflicts of Interest}

The authors have no conflicts to disclose.

\section{REFERENCES}

1. Cahill L. Why sex matters for neuroscience. Nat Rev Neurosci 2006;7:477-484.

2. Afifi M. Gender differences in mental health. Singapore Med J 2007:48:385-391.

3. American Psychiatric Association. Diagnostic and statistical manual of mental disorders. 5th ed. Arlington: American Psychiatric Association, 2013

4. Miller A, Fox NA, Cohn JF, Forbes EE, Sherrill JT, Kovacs M. Regional patterns of brain activity in adults with a history of childhood-onset depression: gender differences and clinical variability. Am J Psychiatry 2002;159:934-940.

5. Nøvik TS, Hervas A, Ralston SJ, Dalsgaard S, Rodrigues Pereira $R$, et al. Influence of gender on attention-deficit/hyperactivity disorder in Europe-ADORE. Eur Child Adolesc Psychiatry 2006;15 Suppl 1:115-124.

6. Loeber R, Burke JD, Lahey BB, Winters A, Zera M. Oppositional defiant and conduct disorder: a review of the past 10 years, part I. J Am Acad Child Adolesc Psychiatry 2000;39:1468-1484.

7. Munkvold LH, Lundervold AJ, Manger T. Oppositional defiant disorder gender differences in co-occurring symptoms of mental health problems in a general population of children. J Abnorm Child Psychol 2011;39:577-587.

8. Forouzan E, Cooke DJ. Figuring out la femme fatale: conceptual and assessment issues concerning psychopathy in females. Behav Sci Law 2005;23:765-778.

9. Cale EM, Lilienfeld SO. Sex differences in psychopathy and antisocial personality disorder. A review and integration. Clin Psychol Rev 2002;22:1179-1207.

10. Forth $A E$, Hart SD, Hare RD. Assessment of psychopathy in male young offenders. Psychol Assess 1990;2:342.

11. Forth AE, Mailloux DL. Psychopathy in youth: what do we know? In: CB Gacono, ed. The clinical and forensic assessment of psychopathy: a practitioner's guide. 1st ed. Mahwah: Lawrence Erlbaum Associates, 2000;25-54. 
12. Frick PJ, Bodin SD, Barry CT. Psychopathic traits and conduct problems in community and clinic-referred samples of children: further development of the psychopathy screening device. Psychol Assess 2000;12:382-393.

13. Horita M, Takizawa Y, Wada Y, Futamata H, Hashimoto T. Sex differences in EEG background activity: a study with quantitative analysis in normal adults. Rinsho Byori 1995;43:177-180.

14. Wada Y, Takizawa Y, Jiang ZY, Yamaguchi N. Gender differences in quantitative EEG at rest and during photic stimulation in normal young adults. Clin Electroencephalogr 1994;25:81-85.

15. Jung HM, Lee YS, Kim S, Kim SK, Jeong JS, Oh JS, et al. Sex-related differences of EEG coherences between patients with schizophrenia and healthy controls. J Korean Biol Pych 2013;20:166178

16. Langrová J, Kremláček J, Kuba M, Kubová Z, Szanyi J. Gender impact on electrophysiological activity of the brain. Physiol res 2012:61 Suppl 2:S119-S127.

17. Baving L, Laucht M, Schmidt MH. Oppositional children differ from healthy children in frontal brain activation. J Abnorm Child Psychol 2000;28:267-275.

18. Hermens DF, Kohn MR, Clarke SD, Gordon E, Williams LM. Sex differences in adolescent ADHD: findings from concurrent EEG and EDA. Clin Neurophysiol 2005;116:1455-1463.

19. Calzada-Reyes A, Alvarez-Amador A, Galán-García L, Valdés-Sosa M. QEEG and LORETA in teenagers with conduct disorder and psychopathic traits. Clin EEG Neurosci 2017;48:189-199.

20. American Psychiatric Association. Diagnostic and statistical manual of mental disorders. 4th ed. Washington: American Psychiatric Association, 2000

21. Frick PJ, Hare RD. Antisocial process screening device: APSD. 1st ed. Toronto: Multi-Health Systems, 2001.

22. Hare RD. The psychopathy checklist-revised. 1st ed. Toronto: Multi-Health Systems, 1991.

23. Ferrero RG, Ferreri AR. Computed EEG analysis. 1st ed. Buenos Aires: Las Heras, 1995:1-15.

24. Pascual-Marqui RD, Michel CM, Lehmann D. Low resolution electromagnetic tomography: a new method for localizing electrical activity in the brain. Int J Psychophysiol 1994;18:49-65.

25. Szava S, Valdes P, Biscay R, Galan L, Bosch J, Clark I, et al. High resolution quantitative EEG analysis. Brain Topogr 1994;6:211-219.

26. Galán L, Biscay R, Rodríguez JL, Pérez-Abalo MC, Rodríguez R. Testing topographic differences between events related brain potentials by using non-parametric combinations of permutation tests. Electroencephalogr Clin Neurophysiol 1997;102:240-
247.

27. Pesarin F, Salmaso L. Permutation tests for complex data: theory, applications and software. 1st ed. Hoboken: John Wiley \& Sons, 2010;25-45.

28. Genovese CR, Lazar NA, Nichols T. Thresholding of statistical maps in functional neuroimaging using the false discovery rate. Neuroimage 2002;15:870-878.

29. Bennett WG, Korein J, Kalmijn M, Grega DM, Campbell M. Electroencephalogram and treatment of hospitalized aggressive children with haloperidol or lithium. Biol Psychiatry 1983;18:14271440.

30. Selin CL, Gottschalk LA. Schizophrenia, conduct disorder and depressive disorder: neuropsychological, speech sample and EEG results. Percept Mot Skills 1983;57:427-444.

31. Shaheen OO, Abdel Rahman S, Madkour O, Ahmed S. Conduct disorder in Egyptian children. Thesis submitted in partial fulfillment of requirements for the degree of M.D. 1990.

32. Tsuboi T. Seizures of childhood a population based and clinic based study. Acta Neurol Scand 1986; Suppl 110:1-237.

33. Clarke A, Barry R, McCarthy R, Selikowitz M. Age and sex effects in the EEG: differences in two subtypes of attention-deficit/hyperactivity disorder. Clin Neurophysiol 2001;112:815-826.

34. Calzada-Reyes A, Alvarez-Amador A, Galán-García L, Valdés Sosa M. EEG abnormalities in psychopath and non-psychopath violent offenders. J Forensic Leg Med 2013;20:19-26.

35. Knyazev GG. EEG delta oscillations as a correlate of basic homeostatic and motivational processes. Neurosci Biobehav Rev 2012;36:677-695.

36. Rangaswamy M, Porjesz B, Chorlian DB, Choi K, Jones KA, Wang K, et al. Theta power in the EEG of alcoholics. Alcohol Clin Exp Res 2003;27:607-615

37. Bauer LO, Hesselbrock VM. Brain maturation and subtypes of conduct disorder: interactive effects on P300 amplitude and topography in male adolescents. J Am Acad Child Adolesc Psychiatry 2003;42:106-115.

38. Éismont EV, Pritchenko OV, Pavlenko VB. Peculiarities of the pattern of EEG activity in institutionalized children. Neurophysiology 2014;46:415-442.

39. Hari R, Salmelin R, Mäkelä JP, Salenius S, Helle M. Magnetoencephalographic cortical rhythms. Int J Psychophysiol 1997;26:5162

40. Fairchild G, Passamonti L, Hurford G, Hagan CC, von dem Hagen EA, van Goozen SH, et al. Brain structure abnormalities in early-onset and adolescent-onset conduct disorder. Am J Psychiatry 
2011;168:624-633

41. Fairchild G, Toschi N, Hagan CC, Goodyer IM, Calder AJ, Passamonti L. Cortical thickness, surface area, and folding alterations in male youths with conduct disorder and varying levels of callous-unemotional traits. Neurolmage Clin 2015;8:253-260.

42. Ermer E, Cope LM, Nyalakanti PK, Calhoun VD, Kiehl KA. Aberrant paralimbic gray matter in incarcerated male adolescents with psychopathic traits. J Am Acad Child Adolesc Psychiatry 2013;52:94-103.

43. Blair RJR. The amygdala and ventromedial prefrontal cortex: functional contributions and dysfunction in psychopathy. Philos Trans R Soc Lond B Biol Sci 2008;363:2557-2565.

44. Thatcher RW, Biver CJ, North D. Spatial-temporal current source correlations and cortical connectivity. Clin EEG Neurosci 2007;38:35-48

45. Sheng T, Gheytanchi A, Aziz-Zadeh L. Default network deactivations are correlated with psychopathic personality traits. PLoS One 2010;5:e12611.

46. Juárez M, Kiehl KA, Calhoun VD. Intrinsic limbic and paralimbic networks are associated with criminal psychopathy. Hum Brain Map 2013;34:1921-1930.

47. Gao Y, Glenn AL, Schug RA, Yang Y, Raine A. The neurobiology of psychopathy: a neurodevelopmental perspective. Can J Psychia- try 2009;54:813-823.

48. Blair RJ. Applying a cognitive neuroscience perspective to the disorder of psychopathy. Dev Psychopathol 2005;17:865-891.

49. Kiehl KA. A cognitive neuroscience perspective on psychopathy: evidence for paralimbic system dysfunction. Psychiatry Res 2006;142:107-128.

50. Müller JL, Sommer M, Wagner V, Lange $K$, Taschler $H$, Röder $\mathrm{CH}$, et al. Abnormalities in emotion processing within cortical and subcortical regions in criminal psychopaths: evidence from a functional magnetic resonance imaging study using pictures with emotional content. Biol Psychiatry 2003;54:152-162.

51. Kiehl KA, Smith AM, Hare RD, Mendrek A, Forster BB, Brink J. Limbic abnormalities in affective processing by criminal psychopaths as revealed by functional magnetic resonance imaging. Biol Psychiatry 2001;50:677-684.

52. Passamonti L, Fairchild G, Fornito A, Goodyer IM, Nimmo-Smith I, Hagan CC, et al. Abnormal anatomical connectivity between the amygdala and orbitofrontal cortex in conduct disorder. PLoS One 2012;7:e48789.

53. Baker RH, Clanton RL, Rogers JC, De Brito SA. Neuroimaging findings in disruptive behavior disorders. CNS Spectr 2015;20:369381. 